\title{
Perfil de Sensibilidade aos Antimicrobianos das Infecções Do Trato Urinário Adquiridas em Adultos e Idosos.
}

Lessandra Michelin ${ }^{1}$; Francine Lopes Petry ${ }^{2}$; Guilherme Rasia Bosi ${ }^{3}$;

Eduardo Comparsi ${ }^{4}$; Renata Bettiol ${ }^{5}$; Fernanda de Oliveira Chiaradia ${ }^{6}$

\section{Resumo}

Introdução: Infecção do trato urinário (ITU) é uma das infecções mais frequentes encontradas em ambiente hospitalar e comunitário. Dentre os germes, os resistentes a multidrogas estão cada vez mais frequentes, conferindo limitação para o tratamento. Para a escolha do antibiótico no tratamento de ITU deve ser considerado fatores como eficácia, efeitos colaterais e perfil de sensibilidade local aos antimicrobianos.

Objetivo: Analisar o perfil epidemiológico e de resistência bacteriana das ITU em consultas de pronto atendimento de três hospitais de Caxias do Sul, para determinar o melhor esquema terapêutico, enfatizando também a frequência e sensibilidade antimicrobiana das bactérias produtoras de ESBL.

Metodologia: Avaliação retrospectiva do resultado de uroculturas e de prontuários médicos de três hospitais da cidade de Caxias do Sul, entre

\footnotetext{
${ }^{1}$ Médica infectologista, mestre e doutora em Biotecnologia/Microbiologia pela Universidade de Caxias do Sul e Pós-doutorado em Vacinologia pela Université de Gèneve. lemichelin@gmail.com

2 Médica especializada em Medicina Interna pelo Hospital Geral de Caxias do Sul. cinepetry@hotmail.com

3 Médico Residente em Hematologia no Hospital de Clínicas de Porto Alegre. guibosi@hotmail.com

4. Médico especialista em medicina do esporte pelo Instituto de Medicina do esporte, vinculado a Universidade de Caxias do Sul.ecomparsi@gmail.com

${ }^{5}$ Médica Residente do serviço de Medicina Interna do Hospital de Clínicas de Porto Alegre. rbettiol@hcpa.edu.br

${ }^{6}$ Acadêmica de Medicina da Universidade de Caxias do Sul. fer.chiaradia@gmail.com
} 
outubro de 2013 a setembro de 2014. Foram incluídos pacientes maiores de 18 anos, com resultados de urocultura positiva com mais de 100.000 UFC, que consultaram em setor de pronto atendimento com queixa de ITU, sendo excluídas aquelas amostras com mais de duas bactérias identificadas.

Resultados: Foram avaliadas 1214 amostras de urina positiva com quadro compatível de ITU. Escherichia coli estava presente em $65 \%$ da amostra. Para infecção urinária não complicada adquirida em comunidade, o perfil de resistência antimicrobiana foi de 12,8\% para nitrofurantoína, ciprofloxacino $14 \%$ e sultametoxazol/trimetropim com $27 \%$. Em infecção complicada, ceftriaxone apresentou $11 \%$ de resistência, cefalotina $22 \%$ e ciprofloxacino de 38\%. Dentre os fatores de risco significativos para ITU por ESBL adquirida em comunidade estão ITU de repetição, OR 24,6 IC (13,7-44,2), hospitalização prévia, OR 6,04 (IC 3,72-9,82), uso prévio de antibiótico, OR 17 IC $(9,02-32,14)$ e uso de cateter vesical de demora OR 8,12 IC $(4,9-13,4)$. Conclusão: O germe mais incidente em todos os grupos de ITU adquirida em comunidade foi a Escherichia coli. Cefalosporinas de segunda e terceira geração são os antibióticos que melhor cobrem infecção complicada e nitrofurantoína é o melhor para não complicada. Presença de ESBL foi mais frequente em pacientes com ITU complicada, tendo, como principal fator de risco para seu aparecimento, uso prévio de antibiótico, hospitalização prévia, uso de cateter vesical e, com menor intensidade, comorbidades como doença cardiovascular, diabetes e doença pulmonar.

Palavras Chaves: infecção do trato urinário, infecção comunitária, infecção complicada 


\section{Introdução}

As infecções do trato urinário estão entre as mais prevalentes na população afetando, anualmente, mais de 150 milhões de pessoas no mundo todo, sendo responsáveis por altos índices de mortalidade. No ano de 2007, nos Estados Unidos da América, foi a infecção bacteriana mais frequente no ambiente ambulatorial, acarretando 10,5 milhões de atendimentos médicos, constituindo $0,9 \%$ de todas as visitas ambulatoriais. No Brasil, sabe-se que a infecção do trato urinário gera 80 novas consultas médicas para cada 1000 atendimentos.

Infecção do trato urinário pode ser diferenciada em complicada e não complicada. Infecção complicada é aquela associada alterações anatômicas e/ou funcionais do trato urinário ou doenças que interferem com os mecanismos de defesa do paciente, ou seja, fatores que aumentam o risco para infecção ou para falha do tratamento (tabela 1). Nas infecções não complicadas, há envolvimento de indivíduos sem outras comorbidades e sem anormalidades urinárias estruturais ou neurológicas. Dessa forma, uma pielonefrite aguda em um paciente saudável pode ser considerada uma ITU não complicada, enquanto uma cistite aguda pode evoluir com complicações, embora a cistite seja considerada uma ITU não complicada.

Dentre os germes causadores de ITU, Escherichia coli é o mais prevalente, tanto nas infecções complicadas, quanto nas não complicadas. Outros agentes comuns são Klebsiella pneumoniae, Staphylococcus saprophyticus, Enterococcus feacalis, Streptococcus do grupo B, Proteus mirabillis, Pseudomonas aeruginosa, Staphylococcus aureus e Cândida sp. Esse dado é amplamente descrito na literatura.

Em relação ao diagnóstico de ITU, a urocultura, associada ao quadro clínico, permanece como principal ferramenta, uma vez que a urina é considerada estéril. Seu uso estava recomendado principalmente em casos de infecções complicadas, pielonefrites e tratamento antimicrobiano recente. Porém, com o alto índice de infecções resistentes aos antimicrobianos comumente usados, também acaba por ser indicada em infecções não complicadas.

Devido à demora do resultado da urocultura, é frequente a necessidade de utilizar antibióticos de modo empírico. Para essa escolha, fatores como perfil de sensibilidade local, efeitos colaterais, ação sistêmica e eficácia são fundamentais. $O$ tratamento precoce com antibióticos diminui a mortalidade ocasionada pelas ITU. Entretanto, seu uso indiscriminado e inapropriado gera outro problema para a medicina atual: a resistência antimicrobiana. Atualmente essa é a principal causa de falha no tratamento antimicrobiano, gerando aumento nos custos e maior mortalidade.

Dentre os germes causadores de ITU resistentes a múltiplas drogas, as bactérias ESBL são as mais comuns, conferindo limitação para as opções de tratamento. Essa mutação normalmente ocorre no grupo das Enterobactereaceas sp, principalmente em Klebsiella sp e E. coli, porém, vem sendo descrita em outros gêneros, incluindo Citrobacter sp, Serratia sp, Proteus sp, Salmonella sp e Enterobacter sp. Bactérias ESBL são produtoras de enzima betalactamase, capaz de hidrolisar os antimicrobianos 
betalactâmicos, como as penicilinas, cefalosporinas e aztreonan, tornando-se resistentes a essas drogas. Não afetam carbapenêmicos e sua atividade pode ser inibida pelo ácido clavulânico. São um grupo de enzimas codificadas por genes presentes em elementos genéticos móveis, como plasmídeos, transposons e íntegrons, os quais normalmente carregam genes de resistência a outras classes de antibióticos. As enzimas de maior relevância clínica são: TEM (mutantes de temoneira), SHV (variáveis dulfidri) e CTXM (cefotaximase). No momento são mais de 300 variações de ESBL, sendo agrupadas em nove famílias, baseadas na sequência de aminoácidos. TEM e SHV costumavam ser os tipos mais frequentes em infecções hospitalares. Hoje, juntamente com o tipo CTXM, também estão comuns em ambiente comunitário.

Um aumento na prevalência mundial de ESBL vem sendo observada desde 2000. Além de sua disseminação em ambiente hospitalar, há aumento também na disseminação na comunidade. Tal fato tem, como uma das principais hipóteses, o uso indiscriminado de antimicrobianos na população. Há, porém, menção a outras hipóteses na literatura. Uma delas seria em decorrência do aumento no uso de antibióticos, tanto profilático quanto terapêutico, em animais de criação em cativeiro da agroindústria, induzindo resistência em bactérias na comunidade. O primeiro surto de ESBL no mundo ocorreu em 1983 na Alemanha, sendo hoje reconhecido globalmente como uma das maiores causas de infecção hospitalar e comunitária.

Dentre as infecções causadas por ESBL, a infecção do trato urinário é a mais comum. Há descrição na literatura dos possíveis fatores de risco normalmente encontrados para infecção por este tipo de bactéria. Entre eles estão cateter vesical, casa de repouso, hospitalização prévia, procedimento do trato geniturinário e uso prévio de cefalosporinas.

As ESBL distribuem-se de forma diferente geograficamente. Em um estudo realizado na Turquia, entre os anos de 2004 e 2005, foi demonstrada a prevalência de $21 \%$ de E. coli produtora de ESBL. Em outros países da Europa, a taxa de ESBL varia em torno de 8 a $10 \%$.

Esse estudo tem como objetivo identificar o melhor esquema antimicrobiano a ser administrado em pacientes com infecções do trato urinário provenientes da comunidade, segmentando os antibióticos mais indicados para o grupo de infecções complicadas e não complicadas. Em consequência do crescente aumento de ESBL, foram identificados os fatores de risco presentes nos pacientes portadores dessas bactérias que chegam em setores de emergência, com a finalidade de auxiliar na identificação precoce e no tratamento adequado, diminuindo a incidência de morbidade e mortalidade associada. 


\section{Materiais e Métodos}

Estudo descritivo, retrospectivo, sobre o perfil de patógenos isolados em uroculturas. As amostras foram obtidas a partir de consultas do setor de pronto atendimento de três hospitais da cidade de Caxias do Sul/RS, Brasil, no período de outubro de 2013 a setembro de 2014. Para essa análise, foram incluídas amostras de pacientes maiores de 18 anos, que consultaram por queixas urinárias nos setores de pronto atendimento descritos, sendo excluídas as uroculturas com crescimento de dois ou mais microrganismos e exames com menos de 100.000 Unidades Formadoras de Colônias (UFC).

Os hospitais envolvidos foram: Hospital Geral, com atendimento exclusivamente público, Hospital Unimed, com atendimento exclusivamente privado e Hospital Pompeia, com atendimento público e privado. Apesar de serem instituições diferentes, os resultados fornecidos de crescimento bacteriano e antibiograma estavam padronizados através de análise automatizada Vitek II ${ }^{\circledR}$. As instituições foram contatadas por documento que esclareceu os objetivos do estudo e assegurou a confidencialidade dos dados obtidos.

A partir das amostras de uroculturas, realizou-se coleta de dados microbiológicos e antibiogramas, assim como revisão dos prontuários para caracterização de fatores de risco para infecção da população estudada. Os itens avaliados encontram-se descritos na tabela 2. A partir dos dados encontrados, os pacientes foram classificados como portadores de infecções complicadas e não complicadas. Para avaliação da idade, houve divisão em subgrupos, conforme recomendação do IBGE (Instituto Brasileiro de Geografia e Estatística), denominandose para grupo 1, pacientes de 18 a 24 anos; grupo 2, de 25 a 54 anos; grupo 3, de 55 a 64 anos e grupo 4, para maiores de 65 anos. Houve também avaliação individualizada da parcela da amostra referente aos portadores de ESBL.

O perfil de resistência de cada antibiótico foi analisado de um modo global, levando-se em consideração todos os germes isolados, com intuito de simular a prática clínica. Em contrapartida, também realizou-se análise de sensibilidade individualizada pelas seis bactérias mais incidentes de cada grupo na amostra, visto que estas representam mais de $90 \%$ de todos os microrganismos isolados. Não foram contabilizados os resultados dos antibióticos com resposta intermediária e aqueles com antibióticos não testados. Para a avaliação do perfil de sensibilidade de infecções, foram utilizados os resultados considerados de primeira escolha para o tratamento, segundo protocolos nacionais e internacionais de $\operatorname{ITU}(3,4,6)$. Sendo assim, para infecções não complicadas, foram avaliados ampicilina, ácido nalidíxico, cefalotina, sulfametoxazol/trimetropim, nitrofurantoína, ciprofloxacino, norfloxacino e gentamicina. Para infecções complicadas, realizou-se comparação com os mesmos antimicrobianos de uso para infecção não complicada sendo, porém, excluídos aqueles antibióticos que não possuem ação sistêmica (nitrofurantoína e norfloxacino). Conforme Takhar (2014), devido às diversas situações que implicam nas infecções urinárias, não 
há consenso sobre qual a melhor terapia a ser utilizada nessas infecções, sendo normalmente recomendadas em algumas situações as mesmas drogas das infecções não complicadas.

Para análise estatística dos dados coletados, foi utilizado média e desvio padrão para as variáveis contínuas. Para variáveis categóricas, foram utilizados percentuais para sua demonstração e, em caso de comparação dessas, utilizou-se o teste qui-quadrado de independência $(\chi 2)$, sendo o resultado apresentado na forma de tabelas e gráficos. $\mathrm{O}$ nível de significância aplicado foi considerado significativo quando $p<0,05$. Para armazenamento do banco de dados e avaliação estatística, utilizou-se o programa IBM SPSS ${ }^{\circledR} 18.0$ para Windows (IBM, Chicago, IL, USA).

O projeto de pesquisa foi analisado pelo Comitê Editorial do Hospital Geral de Caxias do Sul e, por tratar-se de um estudo descritivo, foi dispensado da análise posterior por um Comitê de Ética em Pesquisa após ser aceito o termo de confidenciabilidade de dados.

\section{Resultados}

Mil seiscentos e setenta e quatro exames foram realizados no período proposto. Desses, foram retirados da análise 57 exames em decorrência de os pacientes apresentarem idade menor de 18 anos, 393 por possuírem UFC menor que 100.000 ou presença de 2 germes concomitantes e outros 10 por dados incorretos ou não encontrados, totalizando 1214 exames incluídos no estudo. A amostra foi composta principalmente por mulheres $(76,2 \%)$ e por pessoas de meia-idade $(53,92 \pm 24,03$ anos), sendo 50,1\% acima de 55 anos. O Hospital Pompeia foi responsável pela coleta de 47,5\% dos exames analisados, seguido pelo Hospital Geral de Caxias do Sul com $34,1 \%$. Em relação à classificação das infecções, $47 \%$ da população possui infecção caracterizada como complicada. O sexo masculino é considerado, por si só, infecção complicada, independente dos fatores individuais, conforme literatura $(3,5,6)$. Bactérias produtoras de ESBL foram encontradas em 8,6\% dos casos. As informações gerais da amostra encontram-se descritas na tabela 3 , enquanto as informações referentes à classificação de ITU estão na tabela 4.

Os microrganismos foram avaliados de acordo com a frequencia nos subgrupos de infeção complicada, não complicada e ESBL, conforme detalhado na figura 1. As informações foram agrupadas até o sexto germe mais frequente de cada grupo, devido esta parcela representar acima de $90 \%$ da população estudada. Todos os grupos tiveram Escherichia coli como a bactéria mais frequente (75\% da população geral com infecção não complicada e 50,5\% no grupo de ESBL). Da mesma forma, na população com 
infecção complicada do grupo de ESBL (33,3\%). Klebsiella sp, manteve-se em segundo lugar em todos os grupos e Proteus mirabillis, em terceiro lugar.

$\mathrm{Na}$ avaliação do antibiograma da população geral da amostra, ampicilina foi o antibiótico com o maior número de bactérias resistentes, presente em 62,3\% das infecções complicadas e $47.9 \%$ das não complicadas, seguido por sulfametoxazol/ trimetropim (resistente em 38\% das infecções complicadas e $27,4 \%$ das não complicadas). Outro dado interessante é o fato de ciprofloxacino ser resistente em 38,5\% das infecções complicadas. Esses dados estão nas tabelas 5 e 6.

Para a avaliação dos fatores de risco, foram analisados os dados de pacientes apenas no seu primeiro episódio de infecção urinária, resultando em 842 amostras. Isso se deve ao fato de que, no decorrer do período da análise, muitas pessoas tiveram mais de uma vez diagnóstico de infecção urinária, fazendo com que seu nome ficasse duplicado no banco de dados. As 842 amostras selecionadas para os fatores de risco foram avaliadas para infecção por ESBL em relação à população geral da amostra.

Dentre as associações significativas, ressaltamos nos pacientes portadores de ESBL, o significativo aumento de 24 vezes no risco para desenvolver infecção por germes produtores de betalactamase em pacientes com infecção de repetição e de 17 vezes para esse mesmo desfecho em pacientes com histórico do uso de antibiótico prévio.

Em adultos, a incidência de ITU no sexo feminino é elevada, sendo principalmente relacionada ao início da vida sexual. A mulher possui a uretra mais curta e, devido a isso, é maior a proximidade do ânus com o vestíbulo vaginal e uretra, facilitando a ascensão de bactérias e, consequentemente, maior infecção do trato urinário. No homem, o maior comprimento uretral, assim como o fator antibacteriano prostático, são protetores, sendo esta questão determinante para que a susceptibilidade para ITU na mulher seja maior. Dessa forma, por ter uma anatomia protetora, infecções relatadas no sexo masculino são consideradas como infecção complicada. Os dados obtidos no nosso estudo corroboram com o conhecimento prévio, já que 76,2\% da amostra foi composta por mulheres. No que diz respeito à etiologia das ITU, percebe-se que as bactérias mais frequentemente isoladas nas uroculturas são as enterobactérias, tendo como espécie mais incidente a Escherichia coli, dado já confirmado por outros autores. De acordo com a literatura, tanto infecção complicada quanto não complicada teriam como E. coli o principal germe encontrado, o que também corrobora o achado em nosso estudo.

A avaliação feita, para diferenciar ITU complicada de não complicada, serve para auxiliar o clínico na decisão de nível de atendimento médico necessário (ambulatorial ou hospitalar) e para a escolha apropriada de antibiótico empírico, assim como o tempo de tratamento. No estudo, observou-se que as infecções complicadas compuseram $47 \%$ da amostra, configurando papel importante da escolha de um 
antibiótico empírico adequado, levando em consideração a grande parcela de infecções complicadas e a eficácia das drogas nesses casos.

De acordo com Francesco et al., (2007) e Martins et al., (2010), não se recomenda a utilização de um determinado fármaco na terapia empírica quando a sua taxa de resistência local for superior a $20 \%(35,36)$. Nesse sentido, antibióticos como ciprofloxacino, quando aplicado na população complicada, possuiu taxa de resistência de $38,5 \%$, assim como cefuroxima, que demonstrou resistência bacteriana de 22,6\%. Esses são exemplos de antibióticos comumente usados para tratamento de infecções complicadas que, para a população avaliada nesse estudo, não seria seguro seu uso de forma empírica. Os demais antibióticos comumente usados, como gentamicina, ceftriaxona e cefepime, tiveram taxa de resistência menor que $20 \%(17,7 \%, 11,6 \%$ e $7,9 \%$ ), o que confere a esses antibióticos uma melhor cobertura das infecções complicadas. Há, porém, necessidade de cautela no uso empírico destes, devido possibilidade de resistência antimicrobiana, efeitos colaterais e custo elevado. Ao se tratar de infecção não complicada, os antibióticos que chamam a atenção devido alto grau de resistência são o sulfametoxazol/trimetropim, com taxa de 27,4\% e ampicilina com 62,3\%. Em contrapartida, nitrofurantoína apareceu com taxa de resistência de $12,8 \%$, menor que norfloxacino (15,2\%). Cefalexina apareceu com 1,6\% de taxa de resistência no grupo de infecção complicada e 8,6\% no grupo de infecção não complicada, porém não foi testado em $84 \%$ das amostras, assim como ácido nalidíxico, com taxa de resistência de 4,2\% nas infecções não complicadas e de 3,2\% nas complicadas, porém não tendo sido testado em $91,5 \%$ da amostra. Isso faz com que essa taxa não seja representativa para a amostra.

Os resultados mostraram que, dentre as drogas recomendadas como primeira linha de tratamento empírico para ITU não complicada pela Infectious Disease Society of America e American College of Obstetrician and Gynecologists, o antibiótico com menor perfil de resistência encontrado em nosso estudo é a Nitrofurantoína (12.8\%). Trabalhos recentes vêm reforçando a hipótese de que esta medicação deve ser a droga de escolha para tratamento empírico de ITU não complicada da comunidade, visto que apresenta baixo custo, pequeno impacto na promoção de resistência antimicrobiana e excelente perfil de segurança, tendo como principal inconveniente a necessidade do uso a cada 6 horas durante o dia. Entretanto, com o aumento progressivo na incidência de ITU comunitária por germes multirresistentes, vinha perdendo espaço.

Por outro lado, em relação à infecção complicada, há algumas recomendações indicando o uso de betalactâmicos, dando ênfase para cefalosporinas de $2^{\mathrm{a}}$ e $3^{\mathrm{a}}$ geração, assim como amoxicilina/clavulanato e ampicilina/sulbactam como segunda escolha. Deve, porém, ser guiado por antibiograma assim que possível. Devido aos diversos fatores complicadores e patógenos associados, ainda não há consenso sobre o melhor esquema a ser utilizado. No presente estudo, as cefalosporinas de terceira geração realmente parecem ser eficazes contra as infecções complicadas (ceftriaxona resistente em $11,6 \%$ ), corroborando o que se encontrou na literatura. Já as cefalosporinas de 
segunda geração ficaram com taxa de resistência limítrofe da considerada segura para uso empírico, sendo que cefuroxima foi resistente em 22,6\% das amostras e cefalotina em $22,4 \%$. Outra classe de antibiótico também citado para tratamento dessas infecções, porém com menor eficácia em comparação aos outros já citados, foi as fluoroquinolonas, com exceção daquelas sem ação sistêmica. Em nosso estudo, esses antibióticos não foram considerados seguros para o tratamento empírico devido às taxas de resistência maiores que $20 \%$, como descrito previamente. A prevalência de cepas resistentes às fluoroquinolonas em diversos países da Europa é bastante elevada e varia entre 25 e $50 \%$. Este dado é compatível com os níveis de resistência encontrados em nossa amostra.

Altas taxas de resistência observadas na população geral com ampicilina e sulfametoxazol-trimetropim (37\%) já são previstos, estando em níveis semelhantes aos resultados obtidos por autores de diversas nacionalidades. Este achado é conhecido há muitos anos e torna-se cada vez mais evidente, principalmente em países em desenvolvimento. Sendo assim, seu uso passa a ser desencorajado como tratamento empírico de primeira linha para ITU.

$\mathrm{Na}$ amostra estudada, foi avaliada a presença de germes ESBL, correspondendo a 105 amostras $(8,6 \%)$, dado similar ao encontrado na literatura. A frequencia dos germes encontra-se descrita na figura 1, sendo os mais prevalentes, por ordem de frequência: E.coli, em 35 amostras (40,7\%), seguido de Klebsiella sp, com 28 amostras $(31,4 \%)$ e Enterobacter spp, com 10 amostras (11,6\%). Quando comparado à classificação da infecção, não houve diferença da análise realizada na população total de ESBL. A maior parte das infecções por essa bactéria multirresistente ocorreu no grupo classificados como complicados, estando presente em 86 exames $(81,9 \%)$, contra 19 (18\%) dos exames encontrados nas infecções não complicadas. Nas infecções complicadas notou-se, também, maior frequência dos germes produtores de ESBL em homens em comparação às mulheres (16,3\% e 6,3\% respectivamente). Esses dados sugerem que infecção complicada está associada a uma maior chance de desenvolver germe multirresistente, informação já relatada previamente na comunidade científica.

Há, na literatura, fatores de risco bem descritos para infecção por ESBL. Dessa forma, avaliou-se a população local para comparar esses dados. Os fatores de risco avaliados estão descritos na tabela 7. Alguns, entretanto, apesar de obter relevância estatística, foram considerados não significativos devido ao baixo número de ocorrências na amostra. Exemplo disso ocorreu com a presença de hepatopatia na população estudada. Das 842 amostras, essa doença apareceu em 28 pacientes, desses, 23 possuíam infecção complicada e 18, infecção por ESBL. De acordo com aanálise estatística realizada, o fato de se possuir hepatopatia elevaria a chance em 4 vezes para desenvolvimento de infecção complicada (Odds Ratio 4,44 (1,67 - 11,80) e de 21 vezes para desenvolvimento de infecção por ESBL (Odds Ratio 21,45 (9,5-48,5), com p $<0,0001$. Por se tratar de um número reduzido de hepatopatias avaliadas, esse valor estatístico parece estar muito elevado, em vista que a maior parte dos hepatopatas 
encontrados possuíram infecção complicada e por ESBL. Dessa forma, essa relação não parece condizer com a realidade e com o que é encontrado na literatura. Os fatores referentes a neoplasias prévias e procedimento do trato urinário não foram avaliadas devido grande discrepância entre os dados encontrados pelas múltiplas variáveis, não sendo possível caracterizar como fator de risco sem viés de confusão.

Presença de ITU de repetição é citado na literatura, juntamente com uso prévio de antibióticos, hospitalização prévia e uso de cateter vesical de demora, como um dos principais fatores de risco para desenvolvimento de bactérias multirresistentes. Em nossa análise, sua presença foi vista em 174 vezes das 842 amostras. Comparando essa variável com presença de infecção complicada, vimos que, na amostra desse estudo, há chance de 24 vezes maior de as pessoas com infecção de repetição pertencerem ao grupo de infecção por ESBL. Esse dado não foi encontrado para comparação na literatura, mas certamente contribui para o aparecimento de bactérias multirresistente. Outro dado importante avaliado é a relação de ESBL ao uso prévio de antibióticos. Seu uso estava presente em 258 amostras. Dessas, 82 estão relacionados ao uso de ciprofloxacino, seguido por 61 amostras relacionadas ao uso de ampicilina-sulbactam e 45, a piperacilina/tazobactam. O uso de cefalosporina em geral foi utilizado em 63 vezes, sendo que a maior parte foi utilizada associado ao fator de risco hospitalização prévia. Estudos têm sugerido que a pressão seletiva causada pelo uso constante de cefalosporinas nos centros de saúde contribui para o aparecimento e disseminação desses microrganismos resistentes. Em uma metanálise realizada em pacientes do Canadá e da Turquia, o uso de cefalosporinas prévio aumentou a chance de produzir ESBL em 3,7 vezes. Em nosso estudo, essa variável aumentou a chance em 17 vezes. Como a maior parte da amostra também teve hospitalização prévia, o uso desses antibióticos como fator de risco para o desenvolvimento de ESBL pode ser considerado como um viés de confusão, como também pode ter ocorrido em relação a infecção de repetição.

Hospitalização prévia ocorreu em 331 amostras, aumentando em 6 vezes o risco em se desenvolver ESBL. Na literatura, o dado encontrado foi de aumento em 2,9 vezes. Dessas internações, 85 tiveram passagem em Unidade de tratamento intensivo, sendo associado a uma chance de aumentar o risco de ESBL em 3 vezes. Outra variável bem relacionada com a infecção por ESBL na literatura é a presença de sonda vesical de demora, encontrado nesse estudo com uma chance de 8 vezes em aumentar possibilidade de infecção por ESBL. Conforme Bem- Ami (2009), essa variável aumentou a chance de infecção por ESBL em 4 vezes. Em outro estudo, cateter vesical foi relacionado a $12 \%$ das infecções por ESBL, contra 7 a $10 \%$ em não portadores de cateter vesical. Relacionando as comorbidades, encontrou-se chance aumentada em produzir infecção por ESBL de 4,8 vezes para doença pulmonar e 2,3 vezes para diabetes mellitus. Essas comorbidades estão de acordo com a estatística encontrada na literatura, em que a presença de doença pulmonar aumentou a chance em 2,4 vezes de 
infecção pelo germe resistente, doença cardiovascular em 1,3 e diabetes mellitus em 1,7. Os demais fatores de risco encontrados não demonstraram significância estatística.

Nosso estudo possui algumas limitações. Por tratar-se de um estudo retrospectivo, de análise de resultados de exames já coletados o diagnóstico de ITU foi firmado principalmente baseado no crescimento bacteriano na urocultura, o que pode determinar viés de seleção. Os dados, para determinação de fatores de risco e classificação de infecção, foram coletados retrospectivamente de prontuários e, muitos dados não encontrados, foram considerados como fator negativo, podendo ter subestimada a avaliação. Outra dificuldade encontrada foi em relação a sobreposição de fatores de risco encontrados, não sendo possível, ao certo, avaliar qual fator de risco verdadeiramente influenciou o desfecho. Cientes destas limitações, outras investigações, com melhor padronização das variáveis, mostraram-se pertinentes.

\section{Conclusão}

Levando-se em consideração todos os aspectos levantados, o número da amostra, o período analisado e a rigidez da análise, não há como menosprezar o valor da nitrofurantoína, como droga de primeira linha, no tratamento empírico de ITU não complicada. Em relação ao tratamento das ITU complicada, apesar de mostrar-se como melhor opção o uso de cefalosporinas de terceira geração como tratamento empírico, outros aspectos devem ser avaliados na intenção de evitar uso desnecessário de antibióticos de amplo espectro, o que aumentaria a incidência de bactérias multirresistentes. Torna-se indispensável a individualização de cada paciente quanto a presença de fatores complicadores da ITU, uma vez que presentes, aumentam a chance de fracasso com as drogas comumente utilizadas, entre elas ciprofloxacino, que teve alta taxa de resistência para infecção complicada. Ao analisar a população portadora de germe ESBL, conclui-se que carbapenêmicos firmam-se como principal opção de antibioticoterapia, ainda tendo espaço para o uso de não carbapenêmicos, como a amicacina. É imprescindível, porém, identificar os pacientes com risco para ESBL precocemente a fim de evitar maior mortalidade.

Dos fatores de risco apontados, o uso de cateter vesical de demora e as comorbidades como doença pulmonar e diabetes pareceram influenciar na infecção por ESBL. Os outros fatores de risco, apesar de parecerem influenciar fortemente nessa condição, podem ter se apresentado em sobreposição, o que dificulta a avaliação sobre qual realmente influenciou o desfecho.

O uso de antimicrobianos não deve ser guiado somente pelo mecanismo de ação do microrganismo, mas sim pelo perfil local encontrado (5). Por isso, torna-se imprescindível a realização de estudos demonstrando o perfil de suscetibilidade aos antimicrobianos para adequar o tratamento a realidade local. A caracterização do 
paciente nos grupos de risco auxilia na orientação sobre quem deve ser investigado e também sobre a definição do melhor tratamento empírico a ser utilizado, uma vez que o uso irrestrito de antimicrobianos parecer ser o principal fator para o surgimento de ESBL. Dessa forma, o mapeamento local dos germes envolvidos e suas características de fatores de risco deve ser incentivado, sendo uma das formas de evitar a propagação das bactérias multirresistentes.

\section{Tabela e figuras}

Tabela 1 - Fatores para determinação de infecção complicada do trato urinário

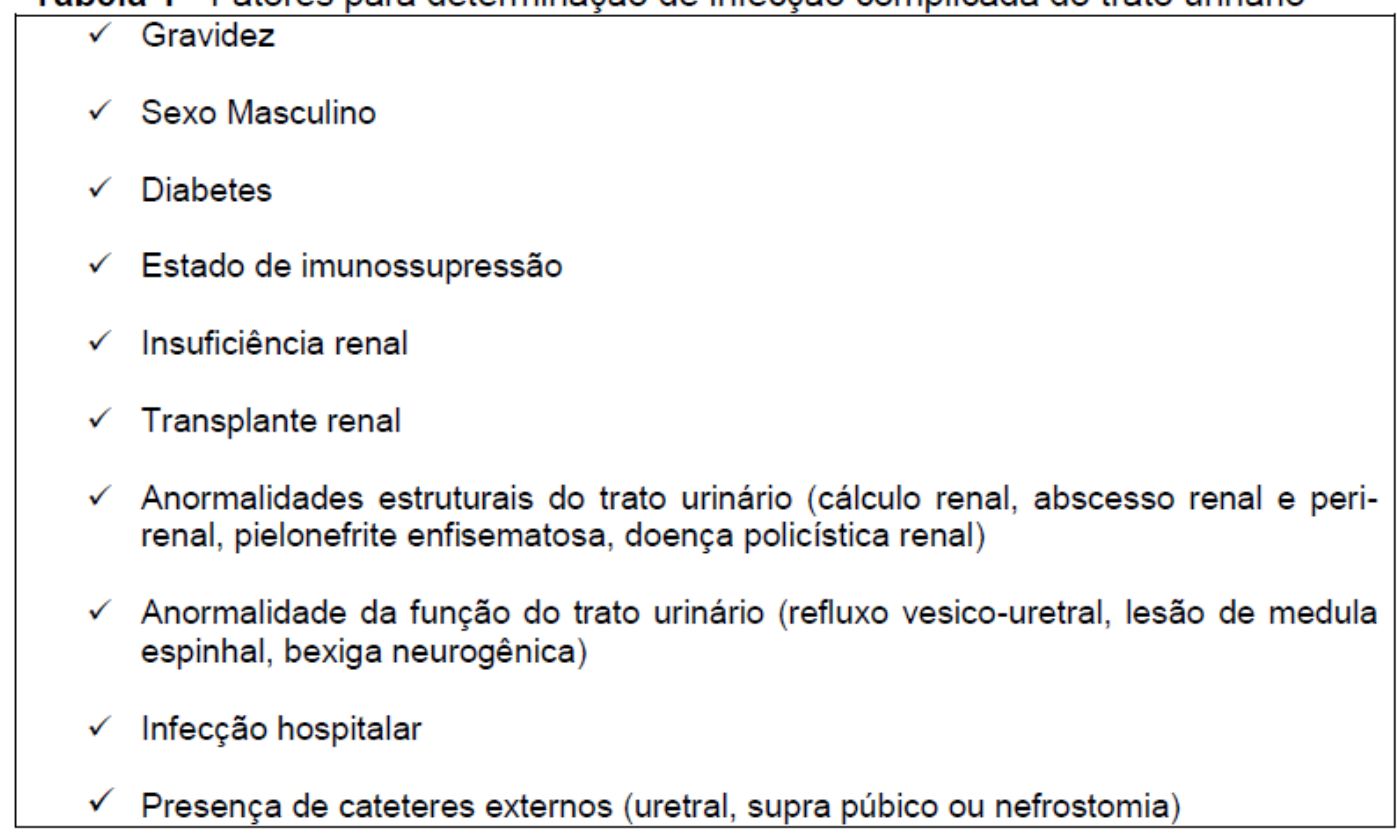

Fonte: Mireles ALF et al. Urinary tract infections: epidemiology, mechanisms of infection and treatment options. Nature Reviews, 2015; 13: 269-84. 


\section{Tabela 2 Dados avaliados na revisão de prontuários}

$\checkmark$ Sexo:

$\checkmark$ Idade;

$\checkmark$ Classificação das ITU entre complicada e não complicada;

$\checkmark$ Doenças Cardiovasculares: Miocardiopatias, infarto agudo do miocárdio, hipertensão arterial sistêmica, doenças valvares;

$\checkmark$ ITU de repetição ( $\geq 3$ episódios ao ano);

$\checkmark$ Hospitalização prévia até 3 meses antes da consulta;

$\checkmark$ Internação prévia em Unidade de Terapia Intensiva até 3 meses;

$\checkmark$ Permanência em casa de repouso;

$\checkmark$ Uso prévio de antibiótico até 3 meses antes da consulta;

$\checkmark$ Procedimento do trato genital: Litotripsia, ressecção transuretral de próstata, prostatectomia, nefrectomia, sling, AMIU, cistolitotomia, cistectomia com reconstrução à Bricker, uretrotomia, perineoplastia, cistectomia e ureteroplastia;

$\checkmark$ Presença de doença renal ou vesical estrutural: como biopsia, duplo $\mathrm{J}$, presença de sonda vesical de demora, refluxo vesico ureteral, litíase renal, hidronefrose;

$\checkmark$ Doença renal crônica;

$\checkmark$ Hemodiálise;

$\checkmark$ Hepatopatia;

$\checkmark$ Pneumopatia;

$\checkmark$ Diabetes Mellitus;

$\checkmark$ Presença de ostomias (gastro, jejuno, traqueo ou cistostomia);

$\checkmark$ Demência irreversivel;

$\checkmark$ Doença sexualmente transmissivel;

$\checkmark$ Gestação. 
Tabela 3. Características gerais da amostra

\begin{tabular}{|c|c|}
\hline Idade (Anos - Média \pm DP) & $53,92 \pm 24,03$ \\
\hline Grupo idade $1(\%)$ & $162(13,3)$ \\
\hline Grupo idade $2(\%)$ & $395(32,5)$ \\
\hline Grupo idade $3(\%)$ & $138(11,3)$ \\
\hline Grupo idade 4 (\%) & $519(42,7)$ \\
\hline \multicolumn{2}{|l|}{ Sexo $(\%)$} \\
\hline Masculino & $289(23,8)$ \\
\hline Feminino & $925(76,1)$ \\
\hline \multicolumn{2}{|l|}{ Laboratório (\%) } \\
\hline Hospital Geral de Caxias do Sul & $434(34,1)$ \\
\hline Hospital Pompeia & $553(47,5)$ \\
\hline Hospital Unimed & $221(18,4)$ \\
\hline
\end{tabular}

DP: Desvio Padrão

Tabela 4 - Classificação das infecções do trato urinário

\begin{tabular}{llll}
\hline & Não ESBL & ESBL & Total \\
\hline Não Complicada & $624(56,2)$ & $19(18,1)$ & $643(52,9)$ \\
Complicada & $485(43,7)$ & $86(81,9)$ & $571(47,1)$ \\
Total & $1109(91,4)$ & $105(8,6)$ & 1214 \\
\hline
\end{tabular}


Figura 1 - Frequência dos germes entre as populações estudadas

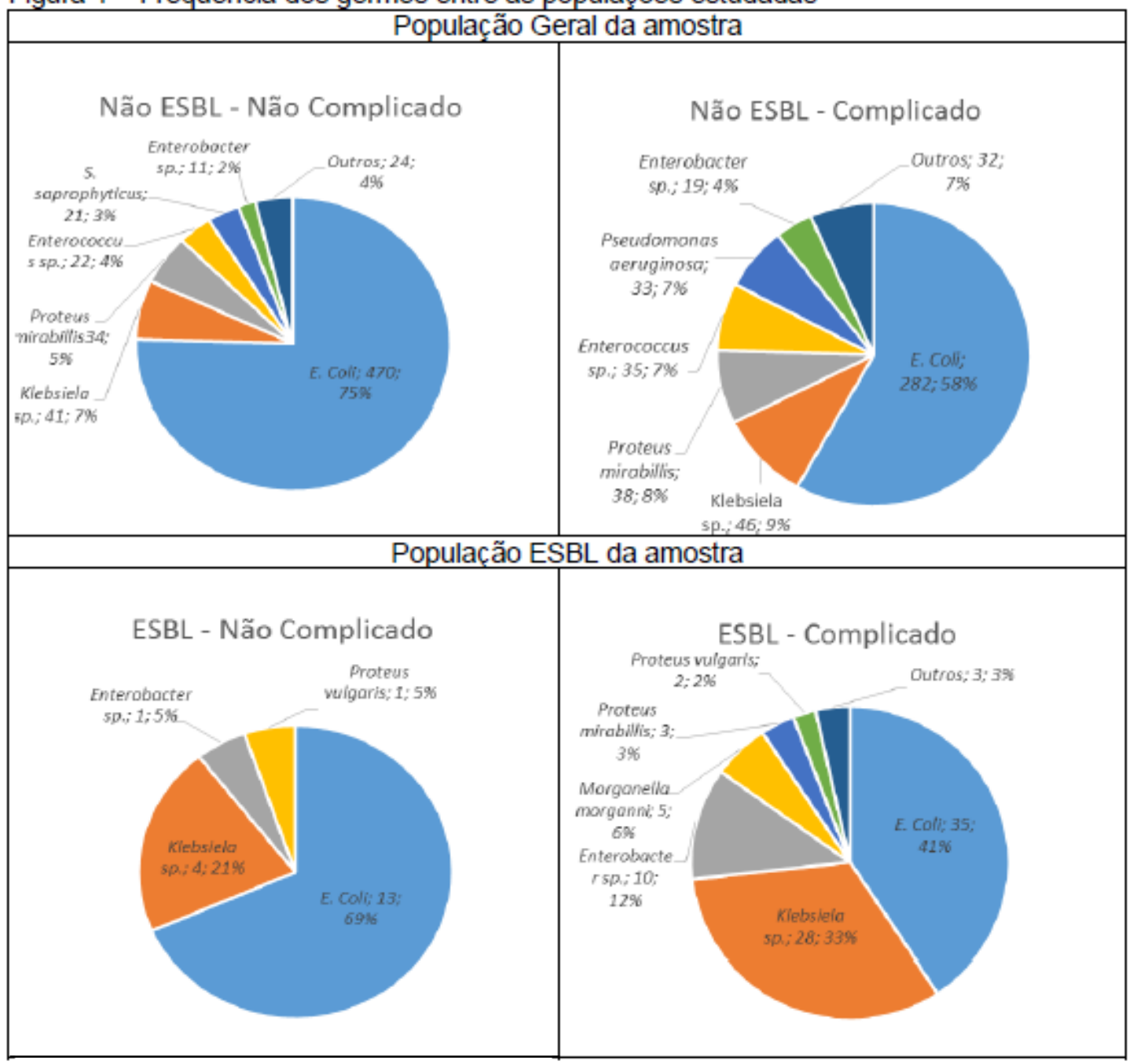


Tabela 5 - Resistência aos antimicrobianos em infecção não complicada.

\begin{tabular}{lc}
\hline \multicolumn{1}{c}{ Antibiótico } & $\begin{array}{c}\text { Não Complicada } \\
\text { (N=643) }\end{array}$ \\
\hline Ampicilina & $308(47,9 \%)$ \\
\hline $\begin{array}{l}\text { Sulfametoxazol/ } \\
\text { Trimetropim }\end{array}$ & $176(27,4 \%)$ \\
\hline Cefalotina & $108(16,8 \%)$ \\
\hline Norfloxacino & $98(15,2 \%)$ \\
\hline Ciprofloxacino & $95(14,8 \%)$ \\
\hline Nitrofurantoína & $82(12,8 \%)$ \\
\hline Cefuroxima & $45(7,0 \%)$ \\
\hline Ac. Nalidíxico & $27(4,2 \%)$ \\
\hline Ceftriaxone & $22(3,4 \%)$ \\
\hline Cefalexina & $10(1,6 \%)$ \\
\hline
\end{tabular}

Tabela 6 - Resistência aos antimicrobianos em infecção complicada.

\begin{tabular}{ll}
\hline \multicolumn{1}{c}{ Antibiótico } & $\begin{array}{c}\text { Complicada } \\
(\mathbf{N}=571)\end{array}$ \\
\hline Ampicilina & $356(62,3 \%)$ \\
\hline Ciprofloxacino & $220(38,5 \%)$ \\
\hline $\begin{array}{l}\text { Sulfametoxazol/ } \\
\text { Trimetropim }\end{array}$ & $217(38,0 \%)$ \\
\hline Cefuroxima & $129(22,6 \%)$ \\
\hline Cefalotina & $128(22,4 \%)$ \\
\hline Gentamicina & $101(17,7 \%)$ \\
\hline Ceftriaxone & $66(11,6 \%)$ \\
\hline Cefalexina & $49(8,6 \%)$ \\
\hline Cefepime & $45(7,9 \%)$ \\
\hline Ac. Nalidíxico & $18(3,2 \%)$ \\
\hline
\end{tabular}


Tabela 7- Fatores de Risco para ESBL

\begin{tabular}{|l|c|c|c|c|}
\hline \multirow{2}{*}{ FATOR DE RISCO } & \multirow{2}{*}{$N^{*}$} & \multicolumn{3}{c|}{ ESBL } \\
\cline { 3 - 5 } & & Frequência & p-value & Odds Ratio $(95 \%$ CI) \\
\hline Gestantes & $93(11,05 \%)$ & $5(5,38 \%)$ & 0,572 & $0,685(0,263-1,78)$ \\
\hline ITU de Repetição & $174(20,67 \%)$ & $64(36,78 \%)$ & $<0,0001$ & $24,6(13,7-44,2)$ \\
\hline Hospitalização & $226(26,84 \%)$ & $\begin{array}{c}162 \\
(71,68 \%)\end{array}$ & $<0,0001$ & $6,04(3,72-9,82)$ \\
\hline UTI & $70(8,31 \%)$ & $16(22,86 \%)$ & $<0,0001$ & $3,22(1,74-5,94)$ \\
\hline Uso Prévio de Antibiótico & $258(30,64 \%)$ & $68(26,36 \%)$ & $<0,0001$ & $17,03(9,022-32,14)$ \\
\hline Doença Pulmonar & $76(9,03 \%)$ & $55(72,37 \%)$ & $<0,0001$ & $4,88(2,78-8,56)$ \\
\hline Doença Renal/Vesical & $85(10,10 \%)$ & $4(4,71 \%)$ & 0,154 & $0,436(0,156-1,223)$ \\
\hline Casa de Repouso & $24(2,85 \%)$ & $5(20,83 \%)$ & 0,118 & $2,6(0,94-7,163)$ \\
\hline IRC & $59(7,01 \%)$ & $5(8,47 \%)$ & 0,936 & $0,861(0,334-2,21)$ \\
\hline Hemodiálise & $28(3,33 \%)$ & $5(17,86 \%)$ & 0,239 & $2,11(0.78-5,71)$ \\
\hline Cardiopatia & $368(43,71 \%)$ & $42(11,41 \%)$ & 0,151 & $1,43(0,90-2,27)$ \\
\hline Hepatopatia & $28(3,33 \%)$ & $18(64,29 \%)$ & $<0,0001$ & $21,457(9,50-48,45)$ \\
\hline Diabetes & $175(20,78 \%)$ & $29(16,57 \%)$ & 0,001 & $2,34(1,44-3,83)$ \\
\hline Ostomias & $26(3,09 \%)$ & $1(3,85 \%)$ & 0,499 & $0,368(0,049-2,75)$ \\
\hline Demência & $58(6,89 \%)$ & $9(15,52 \%)$ & 0,178 & $1,81(0,857-3,84)$ \\
\hline DST & $7(0,83 \%)$ & $2(28,57 \%)$ & 0,287 & $3,82(0,73-20,05)$ \\
\hline Cateter Vesical & $104(34,6 \%)$ & $36(44,4 \%)$ & $<0,001$ & $8,12(4,91-13.45)$ \\
\hline
\end{tabular}

* Total da Amostra: 842 


\title{
Profile of the antimicrobial sensitivity of urinary tract infections acquired in adults and elderly.
}

\begin{abstract}
Introduction: Urinary tract infection (UTI) is one of the most frequently found infections in hospital and community settings. Among the organisms, the ones resistant to multiple drugs are ever more frequent, granting limitations for the treatment. For the choice of antibiotics, it should be taken in consideration factors such as collateral effects and local sensitivity profile.
\end{abstract}

Objective: To analyze the epidemiologic and resistance profile of UTI in emergency consultations of three hospitals in Caxias do Sul to determine the best therapeutic, emphasizing also the frequency and antimicrobial sensitivity of ESBL producing bacteria.

Methods: Retrospective analysis of medical records and urocultures results of three hospitals in Caxias do Sul, between October 2013 and September 2014. Patients at the age of 18 and older were included, with a positive urine culture with more than 100.000 CFU that consulted in emergency department with UTI symptoms, being excluded the samples that had more than two bacteria identified.

Results: 1214 positive urine samples were evaluated all derived from patients with UTI symptoms. Escherichia coli was present in $65 \%$ of the sample. For uncomplicated community acquired UTI antimicrobial resistance profile was $12.8 \%$ for nitrofurantoin, $14 \%$ for ciprofloxacine and $27 \%$ for trimethoprim-sulfamethoxazole. In complicated infections, ceftriaxone presented $11 \%$ resistance, cefalotin $22 \%$ and ciprofloxacin $38 \%$. Among statistically significant risk factors for community acquired urinary infection caused by extended spectrum betalactamase (ESBL) producing enterobacteria are recurrent UTI, OR 24.6 CI (13.7-44.2), previous hospitalization, OR 6.04 (CI 3.729.82), previous use of antibiotics, OR 17 CI (9.02-32.14) and indwelling urinary catheter use OR 8.12 CI (4.9-13.4).

Conclusion: The most frequent organism in all groups from community acquired UTI was Escherichia coli. Second and third generation cephalosporins are the antibiotics that had better cover complicated infections and nitrofurantoin is the best one for uncomplicated infection. ESBL presence was more frequent in patients with complicated UTI, having as major risk factors previous use of antibiotics, previous hospitalization and indwelling urinary catheter use, and as minor risk factors comorbidities such as cardiovascular disease, diabetes and pulmonary disease. 
Keywords: Urinary tract infection, community acquired infection, complicated infection. 


\section{Referências}

1 Picozzi S,Ricci C, Gaeta M. Do we really know the prevalence of multi-drug resistance Escherichia coli in the territorial and nosocomial population? Urol Ann 2013; 5: $25-9$

2. Schappert SM, Rechtsteiner EA. Ambulatory medical care utilization estimates for 2007. Vital Health Stat. 2011; 169:1-38.

3. Mireles ALF, Walker JN, Caparon M, et al. Urinary tract infections: epidemiology, mechanisms of infection and treatment options. Nature Reviews, 2015; 13: 269-84.

4. Dalbosco V, Srougi M, Dall'Oglio M, et al. Infecções do Trato Urinário. Rev Bras Med. 2003;60(6):320-36.

5. Santana TCFS, Pereira EMM, Monteiro SG,et al. Prevalência e Resistência Bacteriana aos Agentes Antimicrobianos de Primeira Escolha nas Infecções do Trato Urinário no Município de São Luis MA. Revista de Patologia Tropical. 2012; 41(4):409-18.

6. Gupta K, Hooton TM, Naber KG, et al. International clinical practice guidelines for the treatment of acute uncomplicated cystitis and pyelonephritis in women: A 2010 update by the Infectious Diseases Society of America and the European Society for Microbiology and Infectious Diseases. Clin Infect Dis. 2011; 52(5):e103-20.

7 Bergeron MG. Treatment of pyelonephritis in adults. Med Clin North Am 1995; 79:619-45)

8. Foxman, B. Urinary Tract Infection syndromes: ocurrence, recurrence, bacteriology, risk factors, and disease burden. Infect Dis. Clin. North Am. 2014; Vol 28: 1-13.

9. Takhar S, Moran GJ. Diagnosis and Management of Urinary Tract Infection in the Emergency Department and Outpatient Settings. Infect Dis Clin N Am 2014; 28:33-48.

10. Wilson ML, Gaido L. Laboratory diagnosis of urinary tract infections in adult patients. Clin Infect Dis. 2004; 38-46.

11. Brown PD, Freeman A, Foxman B. Prevalence and predictors of trimethoprimsulfamethoxazole resistance among uropathogenic Escherichia coli isolates in Michigan. Clin Infect Dis. 2002; 34(8):1061-6.

12. Neal D E. Complicated urinary tract infections. Urol Clin North Am. 2008; Vol 35: $13-22$

13. Abrahamian FM, Krishnadasan A, Mower WR, et al. The association of antimicrobial resistance with cure and quality of life among women with acute uncomplicated cystitis. Infection. 2011; 39: 507-14

14. Cohen ML. Changing patterns of infectious disease. Nature. 2000; 406(6797):762-7 
15. Bauza E, Cercenado E. Klebsiella and Enterobacter Antibiotic resistance and treatment implications. Semin Respis Infecti. 2002; 17:215-30.

16. Zilberberg MD, Shorr AF. Secular trends in Gram-negative resistance among urinary tract infection hospitalizations in the United States, 2000-2009 Infect Control Hosp Epidemiol, 2013; 34. 940-46

17 Bradford PA. Extended-spectrum beta-lactamases in the 21st century: characterization, epidemiology, and detection of this important resistance threat. Clin Microbiol. 2001; 14: 933-51.

18. Minarini LA, Gales AC, Palazzo IC, et al. Prevalence of community-occurring extend-spectrum-Beta-Lactamase- producing Enterobacteriacea in Brazil. Current Microbiology 2007; 54: 335-341

19. Paterson DL, Bonomo RA (2005) Extended-spectrum betalactamases: a clinical update. Clin Microbiol Rev 18: 657-86.

20. Poole K. Resistance to $\beta$-Lactam antibiotics. Cell. Mol. Life Sci. 2004; 61(17):2200-23.

21. Bonnet R. "Growing group of extended-spectrum $\beta$-lactamases: the CTX-M enzymes, "Antimicrobial Agents and Chemotherapy. 2004; 48(1):1-14. [PMC free article] [PubMed]

22. Perez F, Endimiani A, Hujer KM, et al. "The continuing challenge of ESBLs," Current Opinion in Pharmacology. 2007; 7(5):459-69. [PMC free article] [PubMed]

23. Paterson DL, Hujer KM, Hujer AM, et al. "Extended-spectrum b-lactamases in Klebsiella pneumoniae bloodstream isolates from seven countries: dominance and widespread prevalence of SHV- and CTX-M-type b-lactamases." Antimicrob Agents Chemother. 2003; 47:3554-60.[PMC free article] [PubMed]

24. Cho YH, Jung SI, Chung HS, et al. Antimicrobial susceptibilities of extended spectrum beta lactamase producing Escherichia coli and Klebsiella pneumoniae in health care associated urinary tract infection: focus on susceptibility to fosfomycin. Int Urol Nephrol.2005; 47(7):1059-66.

25 ECDC (2011) Annual epidemiological report. European Centre for Disease Prevention and Control (ECDC), Stockholm, Sweden Reporting on 2009 surveillance data and 2010 epidemic intelligence data. Reporting on 2009 surveillance data and 2010 epidemic intelligence data: $183-5$.

26. Leistner R, Meyer E, Gastmeier P, et al. Risk Factors Associated with the Community-Acquired Colonization of Extended-Spectrum Beta-Lactamase (ESBL) Positive Escherichia Coli. An Exploratory Case Control Study. Institute of Hygiene and Environmental Medicine. PLoS ONE 8(9): e74323. 
27. Silva. K, Lincopam N. Epidemiologia das betalactamases de espectro estendido no Brasil: impacto clínico e implicações para o agronegócio. .J Bras Patol Med Lab2012. 48 (2): 91-9.

28. Knothe H, Shah P, Krcmery V, et al. Transferable resistance to cefotaxime, cefoxitin, cefamandole and cefuroxime in clinical isolates of Klebsiella pneumoniae and Serratia marcescens. Infection 1983 11: 315-17. 29. Blanchardièrea.A, Dargère S, Guérin F, et.al. Non-carbapenem therapy of urinary tract infections caused by extendedspectrum $\beta$-lactamase-producing Enterobacteriaceae Médecine et Maladies Infectieuses. 2015; 45(5):169-72

30. Bem-Ami R, Baño JR, Arslan H, et al. A Multinational Survey of Risk Factors for Infection with Extended-Spectrum Beta-Lactamase- Producing Enterobacteriaceae in Nonhospitalized Patients. Clinical Infectious Deseases 2009; 49: 682-90

31. Woerther PL, Burdet C, Chachaty E, et al. Trends in human fecal carriage of extended-spectrum $\beta$-lactamases in the community: toward the globalization of CTX-M. Clin Microbiol Rev 2013; 26:744-52.

32. Heilberg IP, Schor N. Abordagem diagnostica e terapêutica na infecção do trato Urinário (ITU). Rev Assoc Bras Med. 2003; 49(1):109-16.

33. Czaja CA, Scholes D, Hooton TM, Stamm WE, et al. Population-based epidemiologic analysis of acute pyelonephritis. Clin Infect Dis. 2007 Aug 1; 45(3):27380 .

34. Echols RM, Tosiello RL, Haverstock DC, et al. Demographic, clinical, and treatment parameters influencing the outcome of acute cystitis. Clin Infect Dis 1999; 29: 113-19

35. Martins F, Vitorino J, Abreu A, et al. Avaliação do Perfil de susceptibilidade aos antimicrobianos de microrganismos isolados em urinas na Região do Vale do Sousa e Tâmega. Acta Med Port 2010; 23: 641-46

36. Francesco MA, Ravizzola G, Peroni L, et al. Urinary tract infections in Brescia, Italy: Etiology of uropathogens and antimicrobial resistance of common uropathogens. Med Sci Monit 2007; 13: 136-144

37. ACOG Practice Bulletin No. 91: Treatment of urinary tract infections in nonpregnant women. Obstet Gynecol. 2008; 111(3):785-94

38. Munoz-Davila M. Role of Old Antibiotics in the Era of Antibiotic Resistance. Highlighted Nitrofurantoin for the Treatment of Lower Urinary Tract Infections. Antibiotics. 2014; 3(1):39-48.

39. McKinnell JA, Stollenwerk NS, Jung CW, et al. Nitrofurantoin compares favorably to recommended agents as empirical treatment of uncomplicated urinary tract infections in a decision and cost analysis. Mayo Clin Proc. 2011; 86(6):480-8. 
40. Hooton TM. The current management strategies for community-acquired urinary tract infection. Infect Dis Clin North Am 2003; 17:303-32.

41. Wiedemann B, Heisig A, Heisig P. Uncomplicated Urinary Tract Infections and Antibiotic Resistance-Epidemiological and Mechanistic Aspects. Antibiotics 2014; 3(3):341-52.

42. Guneysel O, Onur O, Erdede M, et al. Trimethoprim/sulfamethoxazole resistance in urinary tract infections. J Emerg Med. 2009 May;36(4):338-41

43. Wright SW, Wrenn KD, Haynes MLRN. Trimethoprim-Sulfamethoxazole Resistance Among Urinary Coliform Isolates. Journal of General Internal Medicine. 1999; 14(10):606-9.

44. Raz R, Chazan B, Kennes Y, et al. Empiric use of trimethoprim-sulfamethoxazole (TMP-SMX) in the treatment of women with uncomplicated urinary tract infections, in a geographical area with a high prevalence of TMP-SMX-resistant uropathogens. Clin Infect Dis. 2002 May 1; 34(9):1165-9.

45. Schoevaerdts D, Bogaerts P, Grimmelprez A, et al. Clinical profiles of patients colonize for infected with extended-spectrum beta-lactamase producingEnterobacteriaceae isolates: a 20-month retrospective study at a Belgian University Hospital. BMC Infectious Diseases 2011; 11:12-22

46. Wu UI, Yang CS, Chen WC, et al. Risk factors for bloodstream infections due to extended-spectrum beta-lactamase- producing Escherichia coli. Journal of Microbiology, Immunology and infection 2010; 43: 310-16.

47. Rice, L.B, S H Willey, G A Papanicolaou, et al., Outbreak of ceftazidime resistance caused by extended-spectrum $\beta$-lactamases at a Massachusets chronic-care facility. Antimicrob. Agents Chemother.1990, 34: 2193-9.

48. Spadafino. J, Cohen B, Liu J, et al. Temporal trends and risk factors for extendedspectrum Beta-lactamase-producing E. Coli in adults with cateter-associated urinary tract infections. Antimicrobial Resistance and Infection Control 2014; 3:39-42.

49. Salles M.J.C, Zurita J, Mejía C, et al. Review Article. Resistant Gram-Negative infections in the outpatient setting in Latin America. Epidemiol. Infect. 2013;142:245972. 